\title{
TWT AMPLIFIER USING A FERROELECTRIC CATHODE FOR ELECTRON BEAM GENERATION*
}

\author{
$\underline{\text { Y. Hayashi }}{ }^{\#}$ J. D. Ivers, D. Flechtner, J. A. Nation, P. Wang, Cornell University, Ithaca, NY \\ and S.Banna, L. Schächter, Technion, Haifa, Israel
}

\section{Abstract}

We report first experiments on high-power traveling wave amplifiers using an electron beam generated with a ferroelectric cathode. The electron beam, which is driven by a ferrite core transformer-pulse line system, has energy of $440 \mathrm{keV}$ and a beam current of $40-50$ Amperes. The beam pulse duration is about $250 \mathrm{~ns}$ and the system is operated at $0.07 \mathrm{~Hz}$. The beam is generated from a command switched ferroelectric cathode located in the fringing field of a solenoid. A single stage disk loaded TWT structure is used as the $\sim 9 \mathrm{GHz}$ amplifier. A gain of $10 \mathrm{~dB}$ is observed over the frequency range from 8.9 to $9.4 \mathrm{GHz}$. The main purpose of the experiment is to demonstrate that the ferroelectric generated beam is of good enough quality for microwave amplification in $\mathrm{X}$ band, and to pave the way for use of this cathode in future high power microwave source experiments. We compare the results obtained using this system with the output from a PIC code simulation.

\section{INTRODUCTION}

During the last several years, we have investigated the use of ferroelectric cathodes for electron beam generation. Previous experiments have found that diode currents of up to 330 A could be obtained from a cathode of $\sim 1 \mathrm{~cm}^{2}$ emission area [1]. The beam current in the present design yields $40 \mathrm{~A}$ at $440 \mathrm{kV}$ and will, in the next gun design, be increased to $200 \mathrm{~A}$.

\section{EXPERIMENTAL ARRANGEMENT}

The experimental arrangement is shown schematically in Fig. 1. The cathode gun assembly is connected to the modulator described previously [1]. A more detailed account of the modulator design and the cathode performance in a diode arrangement is to be published shortly [2]. The gun geometry, which was designed using data from the aforementioned diode experiments, uses a planar cathode in a modified Pierce geometry. This geometry produces an approximately uniform cross section beam of $6 \mathrm{~mm}$ diameter throughout the 60 $\mathrm{cm}$ long experiment. The beam is confined by a $\sim 2000$ $\mathrm{G}$ magnetic field generated by the field coils. The $3 \mathrm{~cm}$ diameter ferroelectric cathode is recessed in order to produce a uniform beam with the existing magnetic field geometry. This limits the beam current to $\sim 40 \mathrm{~A}$ at $440 \mathrm{kV}$ in a $250 \mathrm{~ns}$ pulse. The ferroelectric cathode is coated with a $0.2 \mathrm{~mm}$ silver grid on its front surface and a uniform silver layer on its back.. The trigger pulse is adjusted to start slightly before the maximum of the gun voltage pulse, so that when a $\sim 1.5 \mathrm{kV}$ positive trigger pulse is imposed on the back of the ferroelectric cathode, a beam current pulse is obtained. The rise time of the beam current pulse, under appropriate trigger timing conditions, is much shorter than that for the secondary voltage.

The electron gun is connected to an X-band single stage Traveling Wave Tube (TWT), which has 35 uniform

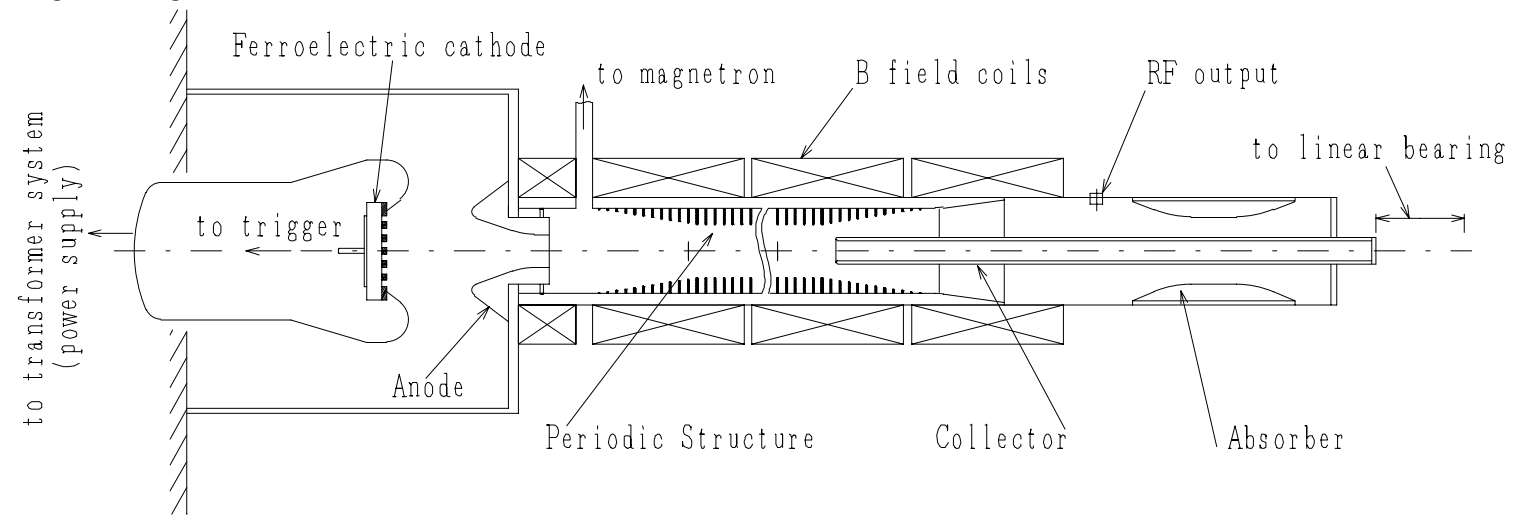

Figure 1: Experimental arrangement for the microwave amplification using a ferroelectric cathode.

"Work supported in by the US Department of Energy and the AFOSR MURI program.

"Email: yh55@cornell.edu 
irises and 11 tapered irises on both ends. The inner and outer radii of the iris are $10 \mathrm{~mm}$ and $16.4 \mathrm{~mm}$ respectively, and the periodic spacing of the irises is $7 \mathrm{~mm}$. This corresponds to the $\pi / 2$ synchronous mode at the operating voltage and at a wave frequency of 9.0 $\mathrm{GHz}$. The input waveguide at the beginning of the TWT is connected to an X-band magnetron which provides $\sim 10 \mathrm{~kW}$ power into the TWT. A reflector mounted on the end of the anode is located at a distance equal to a 3/4 wavelength of the $\mathrm{TE}_{11}$ mode, in order to reduce excitation of this mode. At the end of the TWT, a coaxial mode converter/collector is used to separate the beam and amplified RF output [3]. The beam is dumped into the collector, in order to avoid re-acceleration of the beam electrons by the amplified RF. The amplified $\mathrm{RF}$ is efficiently mode converted from the $\mathrm{TM}_{01}$ mode of the slow wave structure into a TEM mode in the coaxial section. Measurement of the RF output power is made with an E field-probe mounted between the amplifier and the microwave absorber located at the end of the coaxial converter.

\section{EXPERIMENTAL RESULTS AND DISCUSSION}

Figure 2 shows typical waveforms for the gun voltage, the beam current, and the amplified microwave output pulse. The start of diode voltage is defined as $t=0$, and the RF power is taken as the relative power with respect to the magnetron output. At $\mathrm{t}=200 \mathrm{~ns}$, the trigger pulse is applied to the back of the ferroelectric cathode, the current rises to $\sim 40 \mathrm{~A}$ with a rise time much shorter than that of the diode voltage pulse. The pulse width of the current is $\sim 265 \mathrm{~ns}$. The RF pulse rises slightly after the current begins, and continues for up to $250 \mathrm{~ns}$, which is comparable in width to the beam current pulse. A series of experiments have been performed at various RF input frequencies. Fig. 3 shows the amplifier gain and the output pulse width as a function of the frequency. The second scale on the abscissa indicates the cold structure wave phase velocity. The TWT is designed for synchronous operation at a beam energy of $430 \mathrm{keV}$ and a wave frequency of $9.0 \mathrm{GHz}$. In the experiment an RF gain of $\sim 10 \mathrm{~dB}$ was obtained over a frequency range from 8.9 to $9.4 \mathrm{GHz}$. The output gain as predicted by simulation, using the 2D PIC code MAGIC is $16.5 \mathrm{~dB}$ at synchronous flow conditions. This value was obtained however with the center conductor mode converter/beam dump optimally located. In the reported experiments it was retracted. The simulated gain curve lies at lower frequencies by about $250 \mathrm{MHz}$ than the experimental observations. The cold wave phase velocity in the TWT structure changes significantly over the frequency range employed in the amplifier ranging from less than $0.7 \cdot \mathrm{c}$ to $0.87 \cdot \mathrm{c}$ at the lowest frequency. Non-synchronous velocity gain has
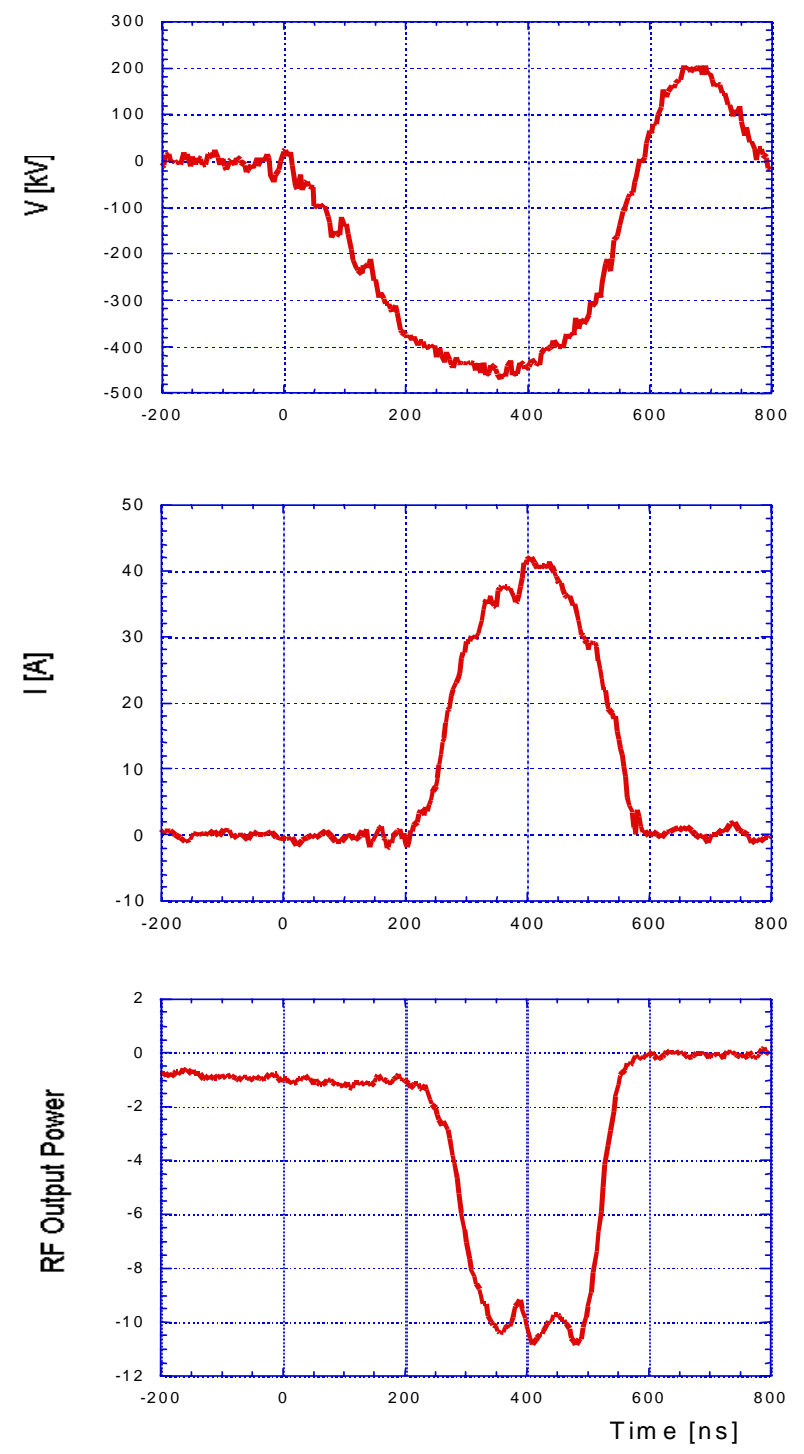

Fig. 2. Waveforms for the electron gun voltage, the beam current, and the microwave output pulse

been previously described and is a relativistic phenomenon [4]. In the simulated data the gain has dropped to zero by $9.2 \mathrm{GHz}$, but gain is seen experimentally with even higher structure wave phase velocities.

An interesting phenomenon is shown in the second part of figure 3 where we indicate that the RF pulse width increases at the higher end of the frequency band to the full beam pulse width. In long, uniform disk loaded structures there is the expectation that the asymmetric HEM modes may develop [5,6,7]. This is particularly likely when the structure inner radius is relatively large compared to the outer radius and the lower branch of the HEM mode overlaps with the TM01 mode passband. The HEM mode will usually lead to beam loss to the wall. In the present case the mode converter at the end of the output section will also act as a 

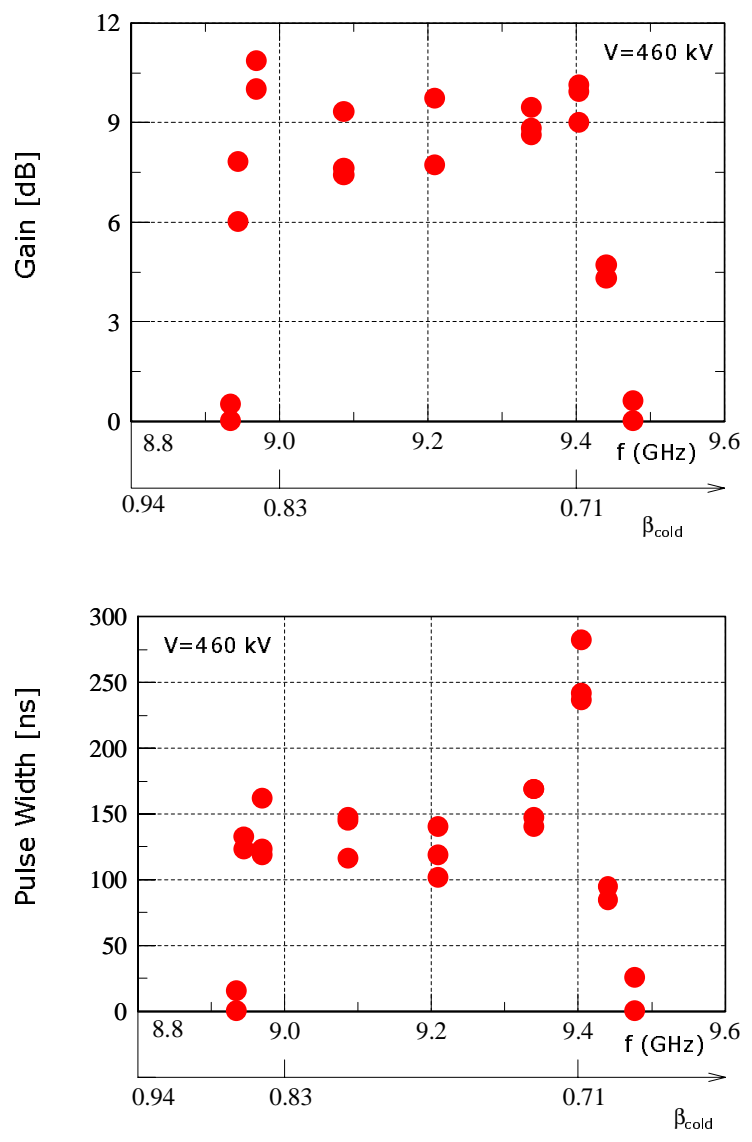

Fig. 3 Characteristics of RF Gain and pulse width vs. frequency.

reflecting boundary for the HEM mode. We have made a preliminary search for the presence of this mode but have not yet identified its presence in these experiments.

In 1992, Shintake [8] proposed the use of choke mode cavities to damp higher-order modes. This approach has also been investigated for use in two beam accelerators [9]. We have performed scalar analyzer measurements of the transmission for a traveling wave structure

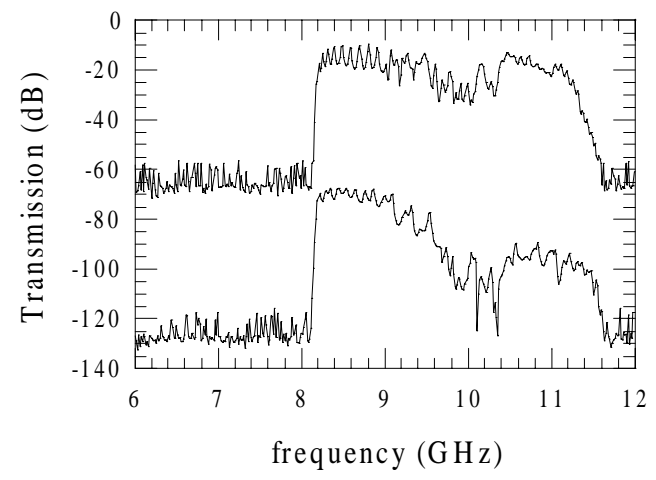

Fig. 4. Comparison of transmission with and without the choke mode cavity. The lower trace is offset by $60 \mathrm{~dB}$. with and without a choke mode cavity. The inner and outer radii of the traveling wave structure are $8 \mathrm{~mm}$ and $14.8 \mathrm{~mm}$ respectively, and the periodic spacing of the irises is $7.5 \mathrm{~mm}$. This structure is identical in dimensions to the uniform part of the amplifier designated as Structure $\mathrm{A}$ in a companion paper presented at this conference [10]. As shown by Figure 4 , the addition of the choke cavity reduced the transmission of the lower $\mathrm{HEM}_{11}$ mode by $\sim 20 \mathrm{~dB}$. Further experiments and theory relating to the excitation of the hybrid mode are reported in the companion paper.

\section{CONCLUSION}

An X-band microwave amplifier using a ferroelectric cathode has been successfully designed and tested. The experimental results mark the first observation of significant microwave amplification from a TWT using a ferroelectric generated electron beam. A $10 \mathrm{~dB}$ gain has been measured over the frequency range from 8.9 to $9.4 \mathrm{GHz}$. The experimental range of frequencies for which gain is observed does not exactly match the simulation result. The reason for this discrepancy is not clear.

A 250 ns wide RF output pulse is obtained at the higher frequencies and some pulse shortening is observed at the lower end of the passband. The pulse shortening might be associated with the onset of excitation of a hybrid mode interaction.

A new electron gun design, which will give the rated beam current of $200 \mathrm{~A}$ at $500 \mathrm{kV}$, is presently under way.

\section{REFERENCES}

[1] J. D. Ivers, D. Flechtner, Cz. Golkowski, G. S. Kerslick, J. A. Nation "A Ferroelectric Cathode,Electron Gun, for High Power Microwave Research", PAC97, 2, 1275-1278, (1998).

[2] J.D. Ivers et al., Accepted for publication in June issue, IEEE Trans on Plasma Physics, (1999).

[3] S.Naqvi, G.S. Kerslick, J.A. Nation, and L. Schachter," Axial Extraction of High Power Microwaves from Relativistic Traveling Wave Amplifiers", Appl. Phys. Letts, 69, 1550, 1996.

[4] S.Naqvi et al., "Resonance shift in Relativistic Traveling Wave Amplifiers", Phys Rev. E, 53, 4229-4231, 1996.

[5] S. Banna, L. Schachter, J. A. Nation, and P Wang, "Beam Interaction with symmetric and Asymmetric Modes in High-Power, High Efficiency, TW Amplifiers", Proc . this Conference.

[6] J.W. Wang and G. Loew, "HEM 11 modes Revisited," LINAC90, LA-12004-C, 135-137, (1990).

[7] J. Haimson and B. Meklenburg, "Suppression of beam induced pulse shortening modes in high power RF generator TW output structures," Intense Microwave and Particle Beams, 209-219, (1992).

[8] T. Shintake, "The Choke Mode Cavity," Jpn. J. Appl. Phys., 31, p. 1567- 1570, (1992).

[9] J.S. Kim, E. Henesteroza, T. Houck, S. Eylon, B.Kulke, G. Westenskow, S. Yu, "RF structure design for the TBNLC," LINAC 96. [10] P. Wang, D. Flechtner, Y. Hayashi, J. D. Ivers, J.A. Nation, X. Zhou, S. Banna and L. Schachter, "A comparative Study of High Power, Multi Stage, TWT X-Band Amplifiers”, Proc . this Conference. 